\title{
Short Gamma Ray Bursts: marking the birth of black holes from coalescing compact binaries
}

\author{
Davide Lazzati ${ }^{1}$ and Rosalba Perna ${ }^{1}$
}

\section{Introduction}

As soon as the catalog of gamma-ray bursts (GRBs) detected by the BATSE (Burst And Transient Source Experiment) had enough events to allow a statistical study, it was discovered that GRB light curves could be separated in two families [44]. Long GRBs are characterized by a duration of more than 2 seconds and a somewhat soft spectrum. Short GRBs, on the other hand, are characterized by a duration of less than 2 seconds and a harder spectrum.

Not much more could be said in the BATSE era, due to the lack of precise localizations and impossibility of long wavelength follow-up that plagued both the long and short GRB populations. With the launch of the Italian-Dutch satellite BeppoSAX, the situation for long GRBs changed dramatically. X-ray, optical and radio afterglows were discovered $[16,87,82]$ to follow the prompt phase. Spectroscopy revealed that the GRBs lie at cosmological distances and that involve explosion energies similar to core collapse supernovae [54, 45]. Evidence of beaming and association to massive stars emerged [72, 77] leading to the now widely accepted scenario of long GRBs as collimated relativistic outflows associated to Type Ib/c supernova explosions [79, 37]. Unfortunately BeppoSAX was non optimally designed to detect short GRBs and none of the above information was available for the short bursts that remained elusive and mysterious. The only advance came from the discovery that short GRBs also have longer wavelength emission on longer timescale [46]. Such discovery was however made on a stacked light curve from past events, and did not allow for any follow-up observation. A general consensus was reached in those years that short GRBs could be associated to the merger of compact binary systems [21], based on a theoretical desire more than on any robust evidence.

JILA, University of Colorado, 440 UCB, Boulder, CO 80309-0440, USA lazzati, rosalba@colorado.edu 
More recently, thanks to the HETE-2 satellite and to Swift, afterglow observations have been performed also for the class of short GRBs, measuring their redshift and energetics, and finally giving some observational corroboration to the idea that they originate from binary mergers [88, 28]. Not everything has been clarified, though, the main problem being now the one of defining what is a long and what is a short bursts, since the two classes seem to have a gray area between them with bursts sharing a complex set of properties. In this review, we critically present the new discoveries made with HETE-2 and Swift, the theoretical advances that were made possible by those discoveries and the still debated issues and future perspectives. This paper is divided in two main sections, the first observational and the second theoretical.

\section{Observations}

\subsection{Pre-Swift era}

Observations performed in the pre-Swift (and HETE-2) era are mainly those performed with BATSE and Konus. Figure 1 shows a histogram of the $T_{90}$ distribution of 2041 GRBs detected by BATSE. The $T_{90}$ is the time interval during which the GRB emits 90 per cent of the total fluence. The solid and dashed lines show the Gaussian fit to the distribution for the short and long bursts, respectively. Even though the bimodality is clear, it is also clear that the two populations are not entirely separated, since a considerable tail of short burst population (about 25 per cent) extends beyond $T_{90}>2 \mathrm{~s}$, the traditional dividing line.

Additional separation between the two classes is provided by the spectral analysis of the light curves. Figure 2 shows in greytones the two dimensional distribution of BATSE GRBs in the hardness-duration plane. The hardness is defined as the ratio of counts in the high energy channels ( 3 and 4) over the counts in the low energy channels (1 and 2). Even though short bursts appear to have a systematically harder spectrum, the two population have a sizable overlap.

The origin of the spectral difference has been analyzed in detail by Ghirlanda et al. [29]. They performed spectroscopy of a sample of 36 bright short bursts comparing the results of several spectral models. They conclude that the spectra of short bursts are successfully fit by a single power-law with an exponential high energy cut-off. They also find that short GRBs have harder spectra due to steeper low energy slopes of the power-law rather than due to a larger perk frequency. It is worth reminding that long GRBs are usually fit wit a smoothly broken power-law model or Band function [3]. 


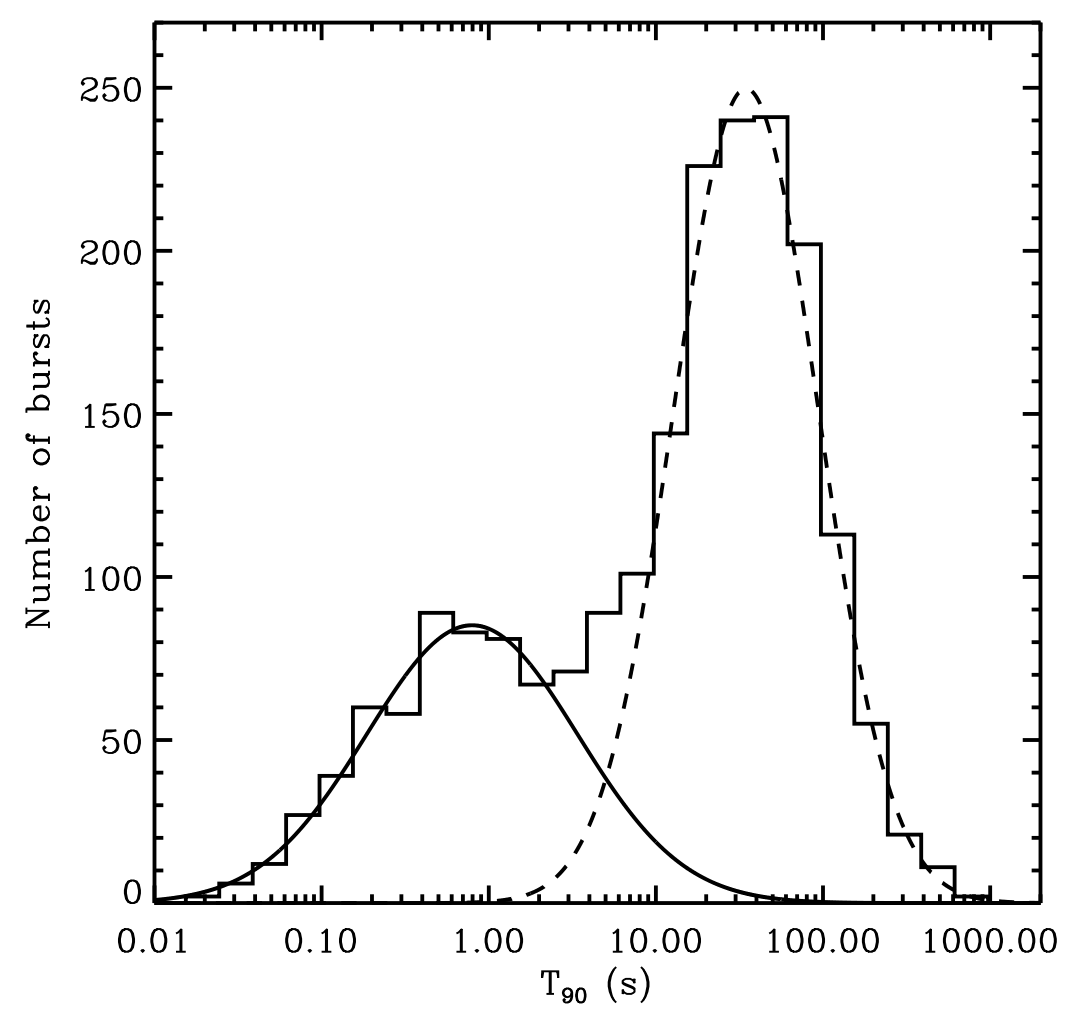

Fig. 1 Distribution of the durations of 2041 BATSE GRBs. Gaussian fits for the short (solid line) and long (dashed line) populations are overlaid.

Nakar \& Piran [56] analyzed the light curves of a sample of short GRBs from BATSE. They find that, when high resolution data are available, short GRB light curves can be resolved into the superposition of many pulses, with statistical properties analogous to those of the long GRBs. This may indicate that the same dynamical and dissipation processes are powering the light curves, even though such processes are still far from being understood.

The quest for short GRB afterglows went on for all the BATSE and BeppoSAX era with very limited results. No afterglow of an individual short GRB was ever found. Lazzati et al. [46] stacked the background subtracted light curve of the 76 brightest short BATSE GRBs looking for an evidence of afterglow in the hard X-rays. They found (see Fig. 3) that there is indeed an excess soft component following the prompt emission of short GRBs lasting approximately 100 seconds. They showed that this component is consistent with being of afterglow origin. As we will see below, it later emerged from 


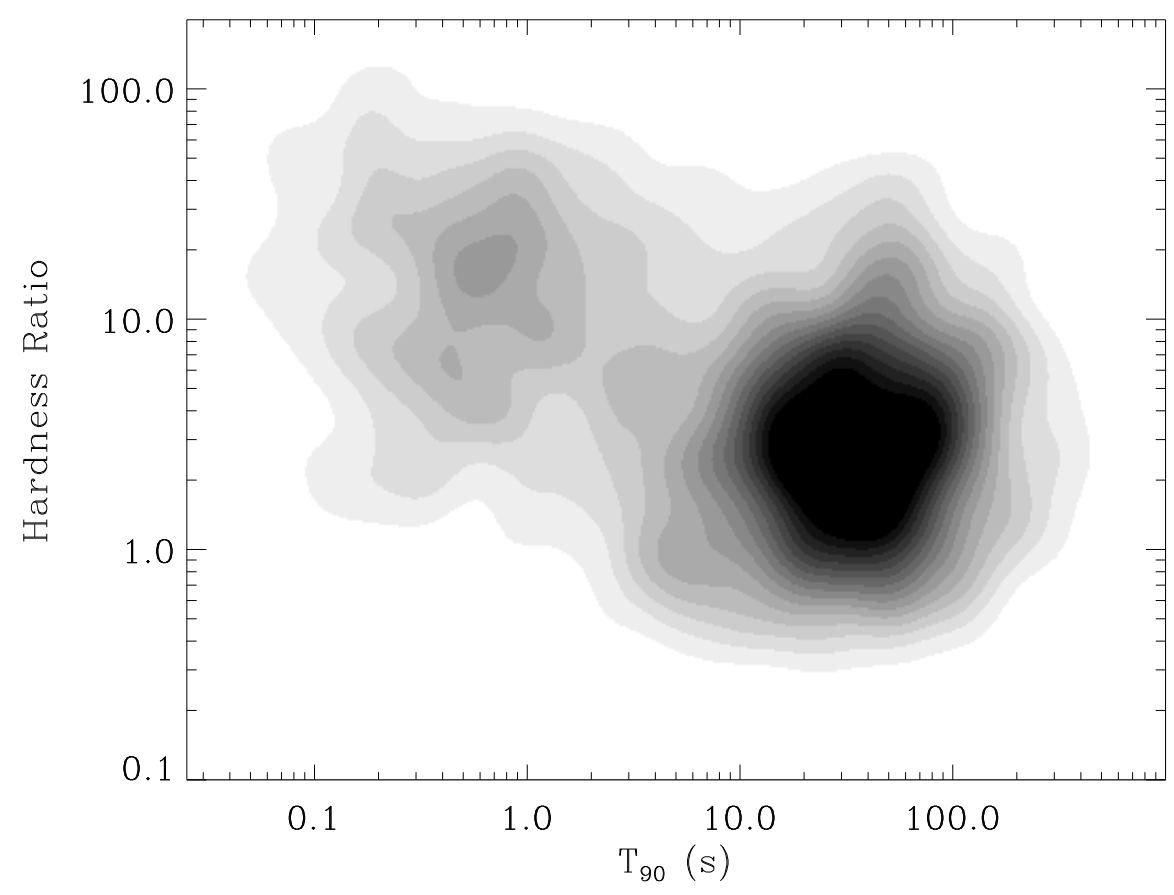

Fig. 2 Hardness-duration distribution of BATSE GRBs.

Swift observations that this component is more likely residual activity from the central engine.

\subsection{The Swift era}

Many of the riddles of short GRB astrophysics have been solved in the Swift era, with a noticeable contribution from the HETE-2 satellite. Due to the characteristics of the BAT detector, Swift observations did not increase our understanding of the prompt emission.

In the spring and summer of 2005, after years of struggle, afterglow of short GRBs were finally detected in X-rays, optical and radio wavelengths $[28,88,14,23,38,17,6]$, bringing the short bursts in the afterglow era.

\subsubsection{Afterglows}

At first sight, afterglows of short GRBs are similar to the afterglows of long GRBs. They are fainter, but qualitatively analogous. Figure 4 shows the X-ray afterglow of GRB 050724 as observed by the Swift XRT [13]. The afterglow 


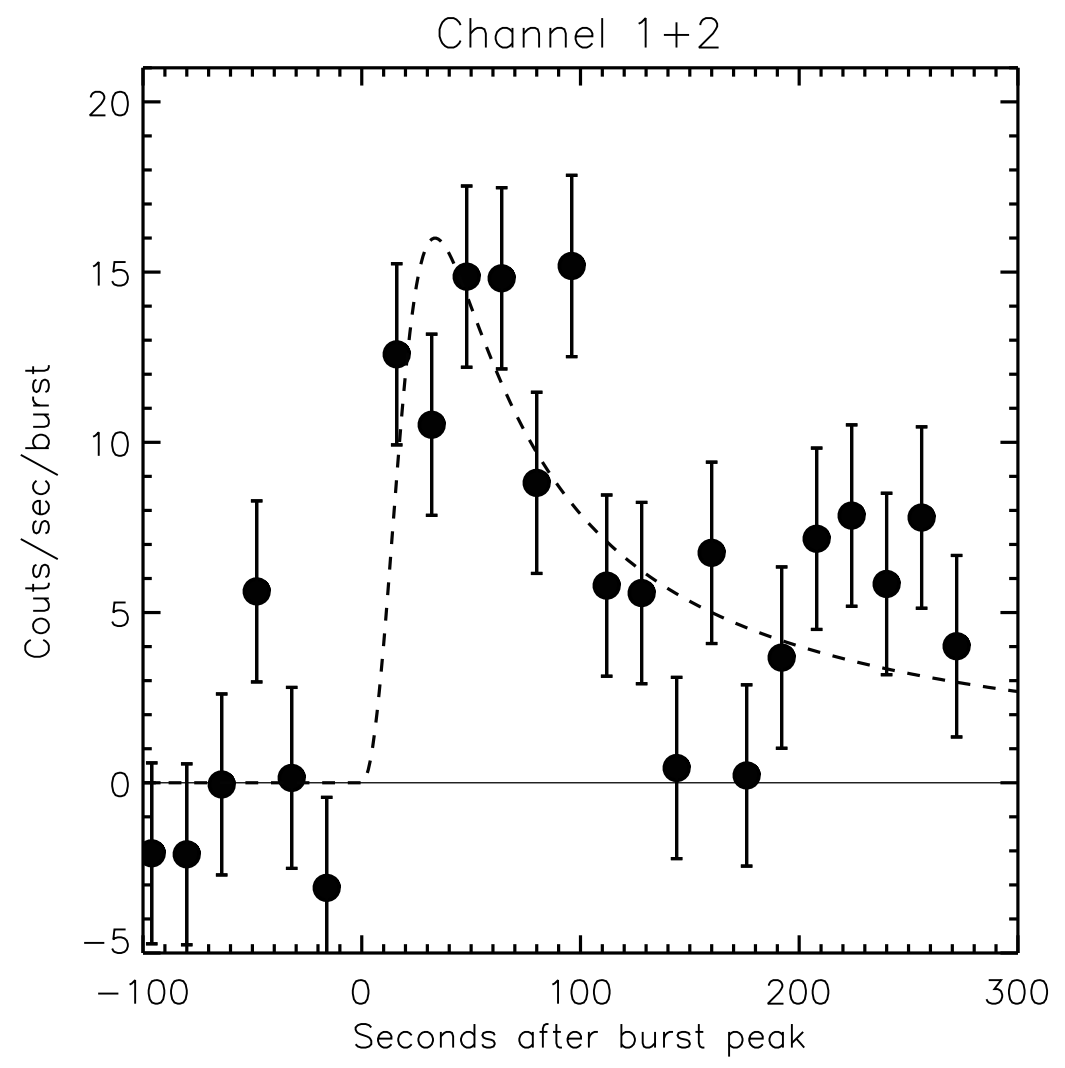

Fig. 3 Light curve of the excess emission found by Lazzati et al, ?? in the composite light curve of 76 bright BATSE short GRBs. The dashed line is a best fit afterglow model.

shows an initially very bright phase, followed by a sharp decline, a possible $t^{-1}$ power-law decay and a flare at late times. The afterglow is overall very faint. This is supposed to be due to a combination of an isotropic equivalent energy smaller than that of long bursts and of a low density interstellar medium, down to $n \sim 10^{-5} \mathrm{~cm}^{-3}$ [58]. Such low densities are thought to be an indication of the binary merger origin of short bursts, since are of the order of magnitude of what expected in the intergalactic medium.

The initial bright phase is likely the one detected on BATSE data [46] and initially interpreted as the regular afterglow. It is now believed to be a sign of continued activity of the central engine (see below). X-ray flares are also common. Differently from the X-ray flares detected on top of long GRB afterglows, the flares so far detected on long short GRBs have long time scale $(\delta t / t \sim 1)$ [49]. Their origin is not clear, a possible detection of rapid variation was reported for GRB 050709 [23], but is inconclusive. Since rapid variations are associate to late time activity of the engine [49], their 
confirmation would be of great relevance for our understanding of the short GRB engine physics.

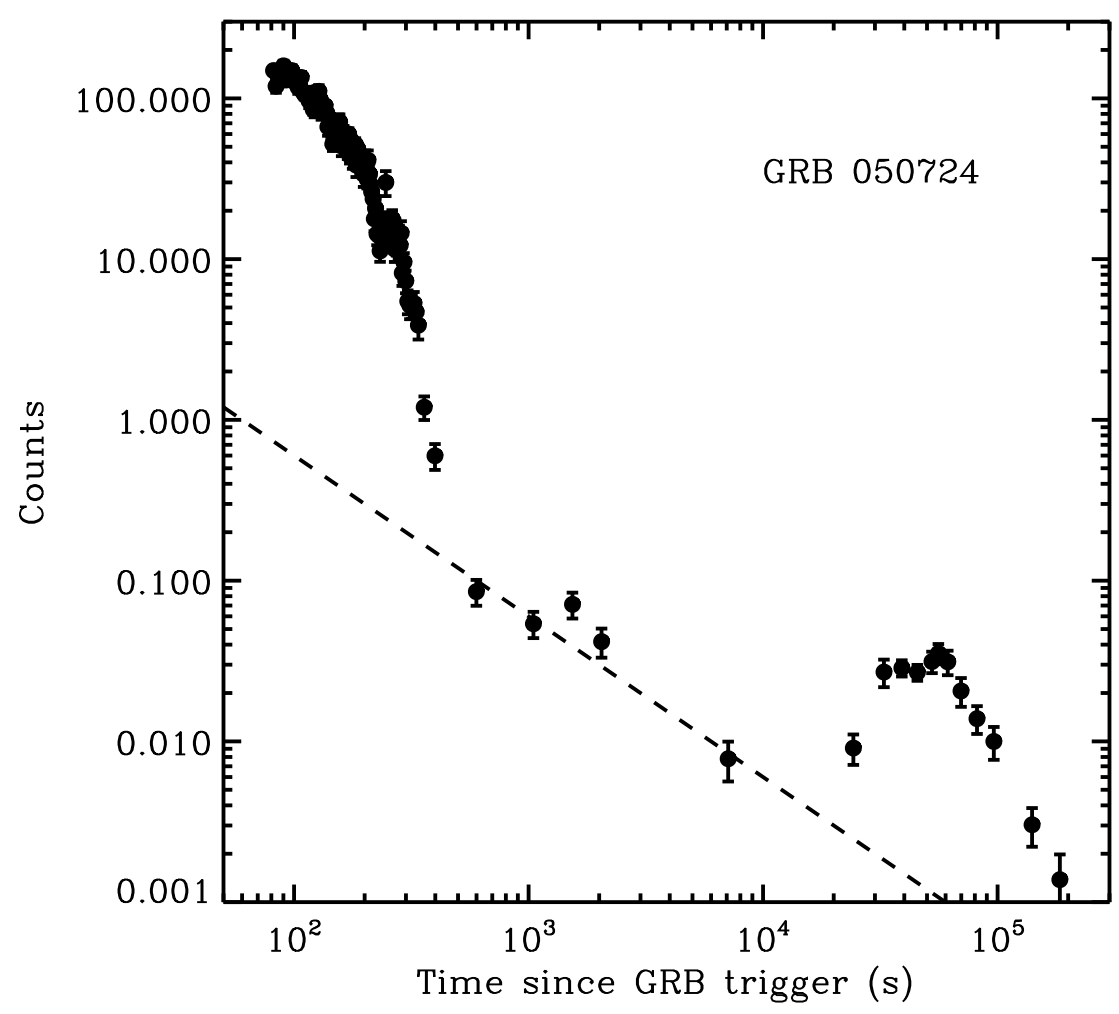

Fig. 4 Swift X-ray afterglow of GRB 050724 [13]. The dashed line shows a $t^{-1}$ power-law for comparison purposes.

It is still debated whether short GRB afterglows show conclusively the presence of beaming of the short GRB fireballs. The question is relevant since the total energy of the explosion depends on the beaming factor. In few cases, a jet break has been claimed. A good example is that of GRB 051221 [78] where a simultaneous break in the optical and X-ray light curves was detected approximately 5 days after the explosion. This is however an isolated case and in most case only an lower limit to the beaming can be obtained [35]. It seems fair to conclude that, even if beamed, short GRBs are less beamed than long GRBs, for which opening angles of few degrees are routinely measured [30]. This observations supports the idea that short GRBs are associated to the merging of compact objects, for which hydrodynamical collimation has a much lesser role than in massive stars [47]. 
Intensive searches for a supernova component have been performed in several short GRBs. Due to their moderate redshift, the searches are very sensitive, being able to detect the presence of a supernova bump even if the supernova was 10 times fainter than the faintest observed supernova [23, 9, 17, 78]. The lack of any detection strongly favors a different progenitor for short and long GRBs.

\subsubsection{Host galaxies}

A debated and important aspect of short GRB research has been that of the identification of the host galaxy. It is important for at least two reasons. First, the identification of a host galaxy is usually the only way to obtain a redshift for short GRBs. Second, host galaxies can provide important clues to the nature of the progenitor of the short bursts and on the physics of their afterglow emission.

Unlike long GRBs, short bursts provided a harder challenge to astronomers for the identification of the host galaxy. While long bursts explode on top of the brightest region of galaxies, making the identification unquestionable [22], short GRBs explode in anonymous regions in the outskirt of galaxies, sometimes having more than one galaxy within their error circles.

The first well-localized short GRB offers a good example of the situation $[39,65,28]$. The X-ray error box was large enough to contain a low-redshift elliptical galaxy, member of a cluster, and several high redshift faint objects, analogous to the host galaxies of long GRBs. The implication of the choice of host were relevant. If the nearby elliptical was the host, short GRBS would be associated to non star forming objects, they would explode in the outskirts of the host - if not outside of them - and would be low redshift events, involving several orders of magnitude less energy than long events. On the other hand, if the progenitor was one of the high redshift objects, short GRBs could be analogous to the long ones. Circumstantial evidence favoring a low redshift origin of short bursts was emerging in the meantime, showing that the location of BATSE short GRBs correlates with local bright galaxies [81] or with clusters [31].

With more short GRB localizations and improvement in the error boxes there is now very little doubt that the population of short GRB host galaxies is markedly different from those of long bursts. Short GRB hosts seem to be a far less homogeneous sample than those of long ones. With the caveat that there may be some misidentifications, given that short GRBs do not explode in the brightest parts of their hosts but rather in their outskirts. Long GRB hosts are usually dwarf starbursting galaxies at moderate to high redshift, always found in the field. Morphologically, short GRB hosts can be of any kind, from early type ellipticals to late type spirals, and are found both in the field and inside clusters. In most cases, short GRB hosts have low star formation, $<1 M_{\odot} / \mathrm{y}^{-1}\left(L_{\star} / L\right)$, or about one hundredth of the star formation 
rate in long GRB hosts [15]. The two populations are different at a very high confidence level [34] and at least in some cases, evidence of an old population of star was found in the host [17, 78]. Approximately 20 per cent of Swift short bursts are associated to clusters of galaxies [7], in agreement with the fraction of stellar mass contained in such systems. Short GRBs seem therefore to be better unbiased samples of the stellar population in the low-moderate redshift universe than long ones.

\subsubsection{Redshift distribution}

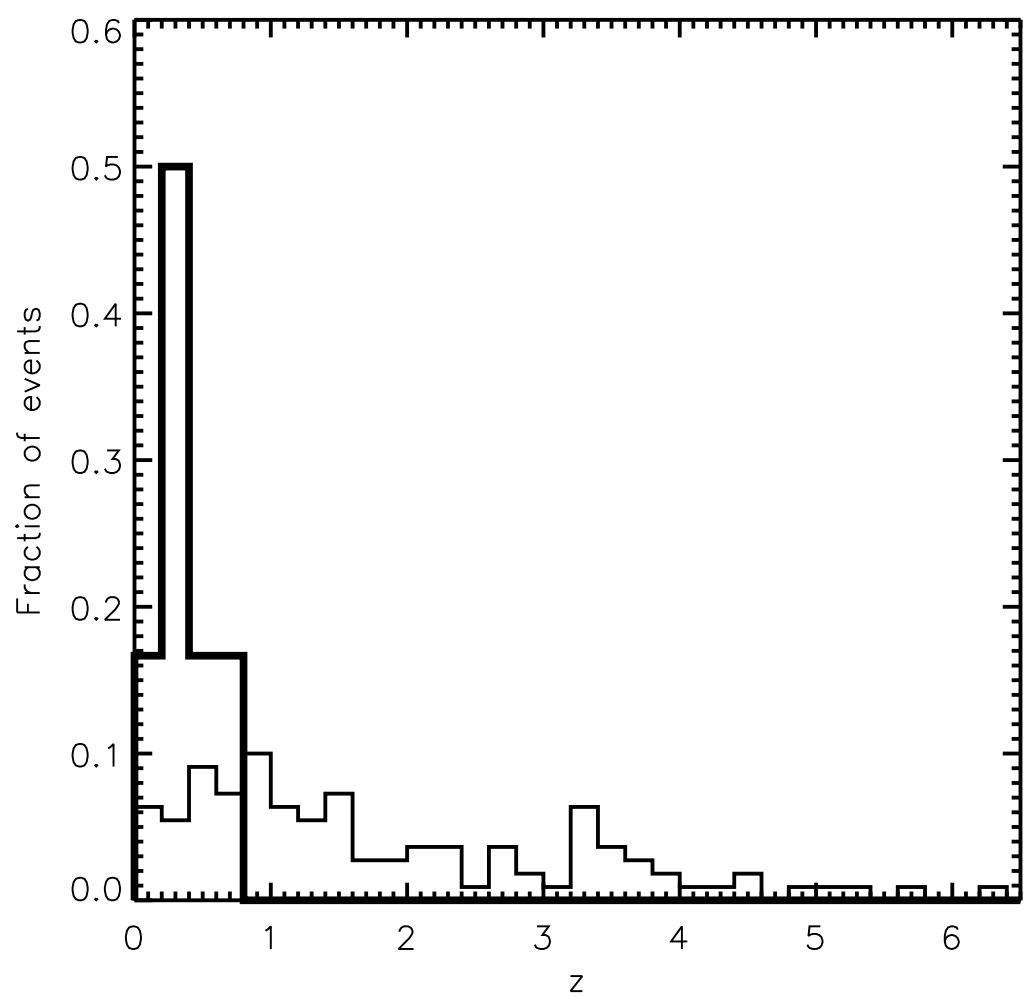

Fig. 5 Redshift distribution of short (thick line) and long (thin line) GRBs.

The redshift of short GRBs is always measured as the redshift of the putative host galaxy and is therefore less robust than the long GRB redshifts, that are often measured both in absorption on the afterglow spectrum and in emission in the host galaxy. Figure 5 shows the short GRB redshift distri- 
bution with a thick line histogram. Even though some of the values can be debated, it is clear that the redshift distribution of short GRBs is centered at much smaller redshifts than that of long GRBs. The long GRB redshift distribution is shown with a thin histogram for comparison and extends beyond redshift $6[80,36,42]$.

\subsubsection{Short or not short?}

We have discusses so far a consistent body of observational evidence coherently supporting the idea that short and long GRBs have different physical origin and are two well separated populations. Long GRBs are long in $\gamma$ rays and soft, have bright afterglows with jet breaks and supernova bumps, they explode in star forming regions inside high redshift dwarf irregular star forming galaxy. Short burst, on the other hand, are short and hard in $\gamma$-rays, have dim afterglows and lack any evidence of an associated supernova, explode in low-intermediate redshift galaxies of all kind, with little sign of star formation.

Such an idyllic interpretation framework was suddenly shaken last summer, when two puzzling bursts were detected: GRB 060505 and GRB 060614 $[19,26,27]$. They appeared to be long in the $\gamma$-ray properties, but had faint afterglows and no sign of an associated supernova, down to very stringent limits. Possible interpretations range from short bursts with a particularly bright X-ray early afterglow [89] to long bursts with a non-massive star progenitor [27], or associated to a SN explosion that did not produce large quantities of ${ }^{56} \mathrm{Ni}[85]$. The puzzle becomes even more intricate if secondary indicators are used. GRB 060614 is found to be consistent with the Amati correlation [1], like a long duration GRB, but to share the short GRB properties in terms of spectral lags (no significant lag was detected) [61].

Even though it is definitely premature to give up a classification successful for decades based on only two events, such observations should clearly ring a warning bell. It is obvious that if short GRBs are followed by relatively bright early X-ray afterglows [46, 13], the distinction between short and long events based only on their duration is band dependent and doomed to fail. However, a new robust scheme has not appeared, so far. Spectral lag are a promising tool, but they are still theoretically unexplained and may well not be associated to the physics of the progenitor, something that seem to be clearly different between the two classes. A classification based on the host galaxy properties is also a possibility that could be considered, with the problem that not all GRBs have host galaxy detections and so many events would become unclassified. It is clear that more events of difficult classification have to be detected before we can establish a new, successful, classification scheme. 


\subsection{SGRs}

A considerable excitement was caused by the detection of a giant flare from the soft gamma repeater (SGR) SGR 1806-20 [64, 83, 12, 52]. The initial phase of the flare is a bright spike lasting less than one second, with a blackbody spectrum and enough energy to be detected by BATSE out to a distance of approximately $50 \mathrm{Mpc}$. It is followed by a pulsed X-ray tail that would be undetectable with any present instrumentation for an extragalactic flare. These properties suggested that a significant fraction of BATSE short GRBs could indeed be giant flares from SGRs in the local universe, out to the Virgo cluster of galaxies. A large effort was attempted to constrain this fraction and possibly identify the extragalactic SGRs in the BATSE sample. The search was based on positional coincidence with nearby galaxies [57,62, 69], on the spectral properties of the prompt emission [48], and on the presence of an oscillating tail. None of these searches found any suitable candidate, implying a fraction of at most 15 per cent of BATSE short GRBs being SGR flares in the local universe. This limit is only marginally consistent with the Galactic SGR rate, and requires the SGR1806-20 flare to be an exceptional event. Of course, caution should always be considered when statistics is based on a handful of events.

\section{Theory}

\section{Binary mergers as progenitors of short GRBs}

The leading candidates as progenitors of short GRBs are mergers of NS-NS binaries $[63,32,21,60]$ and BH-NS binaries [60, 55]. Two compact objects in a binary are bound to eventually merge due to the emission of gravitational wave radiation that causes a loss of energy resulting in a gradual shrinking of the orbit. For typical binary parameters, binaries are expected to coalesce within a Hubble time, as discussed in more detail in the following. From an energetic point of view, there is enough gravitational binding energy that is liberated during the merger to power the GRB and its subsequent afterglow. The engine that helps channeling this energy into a relativistic flow is believed to be, like in the case of long GRBs, an hyper-accreting accretion disk. However, while in the case of long bursts (which are thought to be associated with the collapse of massive stars), the event duration is set by the collapse time of the star envelope (on the order of several tens of seconds) that keeps on feeding the disk, in the case of a binary merger the activity phase is set by the viscous timescale of the disk, which is a fraction of a second, the right order of magnitude needed for the power source of short bursts. 
From an observational standpoint, double neutron stars are known to exist from direct observations in the Galaxy. Well-known examples are the HulseTaylor binary and the binary pulsar [11]. On the other hand, compact-object binaries where one of the components is a black hole have not been observed so far. However, theoretical modeling of binary evolution predicts that binaries with black holes are expected (e.g. [10, 70, 24, 4]). It should be noted that, even if $\mathrm{BH}-\mathrm{BH}$ binaries are also predicted as a possible end state of binary evolution, however they are not expected to give rise to a GRB when they merge. This is because, in this scenario, there would be no fuel to power the accretion disk that could then provide the source of energy for the GRB (see $\S ? ?)$.

In order for mergers of compact objects to be progenitors of short bursts, a fundamental condition is that the merger event rate be comparable to that of the bursts. With the growing number of short bursts with detected redshift, a comparison can be made not only for the overall (i.e. redshiftintegrated) event rate, but also for its redshift evolution. As the sample grows, the calibration of the cosmic rate evolution, in combination with observations of the burst rate as a function of the galaxy type, can allow one to further constrain various types of binary models.

The main source of guidance for the expected characteristics of a population of bursts associated with the coalescence of two compact objects is provided by population synthesis calculations. In the following, we will describe the main assumptions, calculation methods, and results from these calculations (§??), with a special emphasis on their specific application to short bursts (S??). Finally (§??), we will discuss the highlights of numerical calculations that simulate the final moments of the binary, and the formation of the accretion disk that eventually leads to the GRB.

\subsection{Binary evolution - theoretical modeling}

In the last few years, several groups [70,4] have developed population synthesis calculations. These simulations, starting from some assumptions regarding the binary star population, track the evolution of stars both individually and in relation to the companion in the binary system; in output, they are able to predict the fraction of systems that end up with a certain type of compact objects, the merger time of each binary, and hence the cosmic event rate. In the following, we describe in more detail the highlights of the population synthesis calculations.

The evolution of each star in the binary system is computed given its zeroage main-sequence mass and its metallicity. The code tracks all the stages

from the main sequence to the red giant branch to the core helium burning and the asymptotic giant branch. At each stage of evolution, the basic stellar parameters (radius, luminosity, stellar mass, core mass) are determined. 
While each star is evolved depending on its initial parameters, a number of effects that influence the binary orbit (e.g. mass and angular momentum losses due to stellar winds) are taken into account. At every evolutionary time step, the codes check for possible binary interactions. If any of the components fills its Roche lobe, then the resulting mass transfer is computed, and so the eventual resulting mass and angular momentum losses. If the binary survives the mass transfer event (i.e. both stellar components fit within their Roche lobe), then the evolution of the binary keeps on being followed. The calculation for each star ends at the formation of a stellar remnant: a white dwarf, a neutron star or a black hole. If the remnant is born with a supernova explosion, the effects of the supernova kicks and mass loss on the binary orbit are computed. Finally, once a binary consists of two remnants, its merger lifetime is calculated, i.e. the time until which the components merge due to emission of gravitational radiation and the consequent orbital decay.

Early population synthesis studies $[70,25]$ found that, for the NS-NS binary systems, merger times are generally long, $t_{\text {merg }}>0.1-1$ Gyr. Smaller timescales were found [86] as a result of the assumption that the secondary star, once it becomes a low-mass helium-rich star, can initiate an extra mass transfer phase. More recently, simulations [4] identified a new population of coalescing NS-NS binaries, which merge on a timescale which is much shorter than that of the 'classical" population. These very short timescales, $t_{\text {merg }} 0.001-1 \mathrm{Myr}$, are the result of allowing both the primary and the secondary star to initiate an extra mass transfer phase. Furthermore, the inclusion of natal kicks in the simulations [4] further contributes to decrease the merger timescales. Accounting for natal kicks, in fact, results in the disruption of the widest binaries and in an eccentricity gain for the remaining ones, which further reduces their lifetimes. This subpopulation of relatively short-lived NS-NS binaries was found to be the dominant channel, making up about $80 \%$ of the total population.

On the other hand, all population synthesis calculations agree on the typical distribution of merger times of the NS-BH systems, which is found to be similar to that of the "classical" population of NS-NS binaries, i.e. $t_{\text {merg }}>0.1-1$ Gyr.

\subsection{Theoretical predictions for the observational properties of short bursts due to binary mergers}

a) Rates The distribution of merger times obtained from population synthesis calculations, combined with a prescription for the cosmic star formation rate, allows one to estimate the rate of binary mergers throughout the lifetime of the Universe. Since the simulations by Belczynski et al. [5] include the population of tight NS-NS binaries (i.e. the short-lived population), the predicted cosmic redshift rates are different for the population of NS-NS and 
of NS-BH binaries. An example is shown in Figure 1, with data from the simulations by Belczynski et al. [5]. Those calculations used as star formation rate which peaks at a redshift of about 3 and has only a mild decline up to redshifts of about 5 . Due to the merger time delays, however, the predicted binary merger rate peaks at a remarkably lower redshift. The larger the time delay, the larger the shift to lower redshifts of the binary merger event rate compared to that of the underlying star forming rate. This is especially emphasized in Figure 1, where the rates due to the fraction of (both NS-NS and NS-BH) long-lived binaries $(t>100 \mathrm{Myr})$ are shown together with the overall rates. Whereas the assumed star-formation rate remains constant up to a redshift of $\sim 5$, the rate of the long-lived component drops substantially. For the overall binary merger rate, if the assumed SFR peaks at a redshift of $\sim 3$, the peak is found in the range $\sim 1-2$.

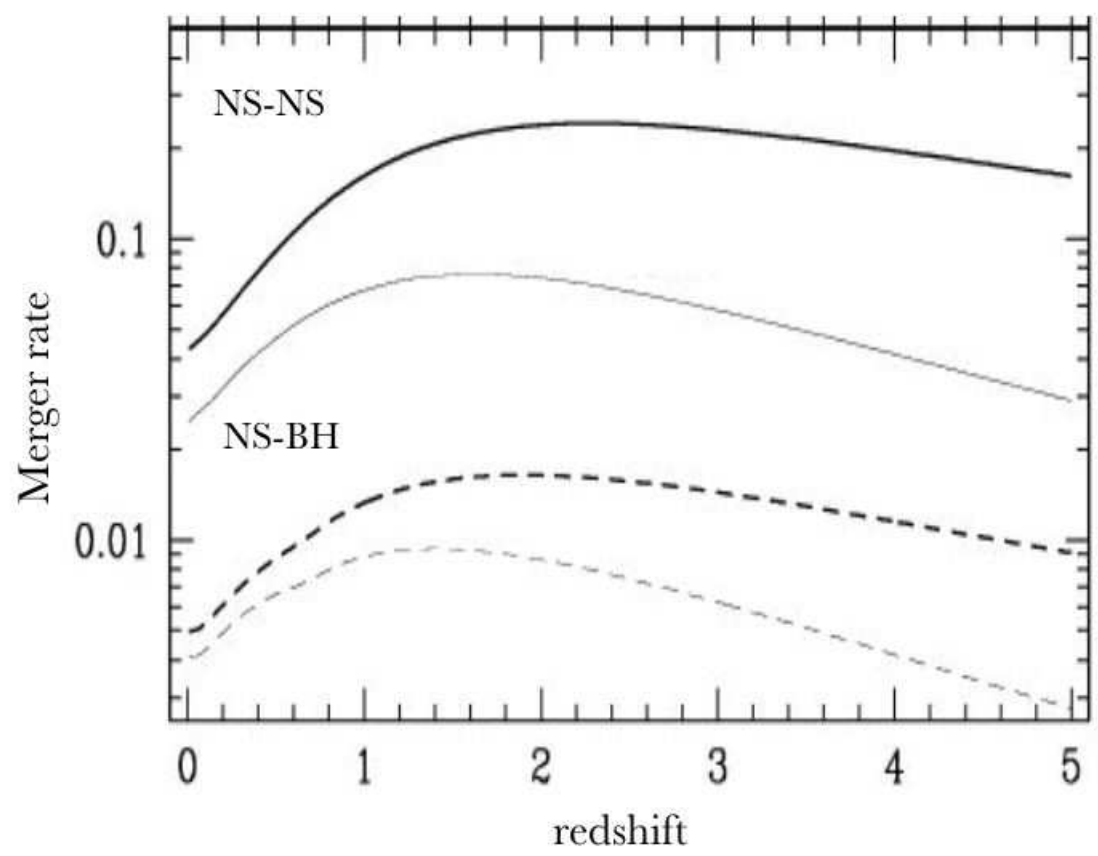

Fig. 6 Thick lines: the inferred merger rate as a function of redshift for the NS-NS and BH-NS mergers. Thin lines: the contribution from the long-lived $(t>100 \mathrm{Myr})$ population. Rates are in arbitrary units. Data from the simulations of Belczynski et al. [5].

Another method of estimating the binary merger rates starts from a count of the observed number of NS-NS binaries in the Milky Way. This number is then corrected to account for the completeness of the survey and transformed into a local rate by weighing in the estimated lifetimes of the observed systems. Up to date, the latest calculations of the Galactic merger rates [41] 
have yielded a value in the range $1.7 \times 10^{-5}$ to $2.9 \times 10^{-4} \mathrm{yr}^{-1}$ at the $95 \%$ level.

em b) Offsets from host galaxies, densities of the circumburst medium, afterglow brightnesses and galaxy types

Since the first studies on GRBs, it was clear that the location of a burst within a galaxy, and hence its environment, would provide important clues to the properties of the progenitor itself. In the past decade, a number of groups $[25,8,66,5]$ have performed statistical studies of the distribution of the binary merger sites in a galaxy. Generally speaking, progenitors with very short lifetimes are typically expected to produce GRBs close to their place of birth. However, if the progenitor is endowed with a kick velocity (such as in the case of binaries), then the merger sites could have a substantial offset from the place of birth. The amount of the offset will generally depend on a combination of the merger time and the velocity of the binary system, as well as the potential well in which the systems move. Kick velocities are estimated to be in the range of several tens to several hundreds $\mathrm{km} / \mathrm{s}$ [40]. A binary with a velocity in the upper range of the distribution, and a long lifetime $\sim$ a few Gpc, can travel a substantial distance, on the order of the Mpc scale, before merging. On the other hand, for binaries with a short lifetime, on the order of a million years or less, the place of merger is always going to be close to the place of birth even for the fastest pulsars of the distribution. Therefore, it is clear that the expectations for the distribution of the merger sites are going to heavily depend on the expected distribution of merger times of the binary population under consideration.

Another element of consideration is the fact that different types of galaxies are expected to host different types of stellar populations, with, at one extreme, starburst galaxies which are dominated by very young stars, and at the other end, elliptical galaxies which have almost no star formation rate at all and hence are dominated by an old stellar population. These different types of galaxies, therefore, will select out different components of the binary mergers. Starburst galaxies are expected to be dominated by the short-lived binary population, while elliptical galaxies will mostly host the merger events of the long-lived population. Spiral galaxies are in between, since they host both young and hold star populations. As a result of these differences, merger events taking place in elliptical galaxies will generally occur at larger offsets (from the galaxy centers) with respect to merger events that occur in starburst and spiral galaxies. Furthermore, within a given morphological galaxy type, mergers in small galaxies will have substantially larger offsets than merger in large galaxies. This is due to the larger gravitational potential of the larger galaxies, which prevents the binaries from traveling too far.

The sites of the binary mergers are especially important in determining the observability of the GRB afterglows. Since the gas density declines with the distance from the galaxy center, mergers that occur in the outskirts of galaxies are expected to give rise to very dim afterglows. Based on the considerations above, short bursts occurring in large starburst galaxies are expected to be the 
brightest, while short bursts occurring in small ellipticals will be generally the dimmest. A fraction of these bursts are expected to be "naked", i.e. completely lacking any afterglow emission, especially at wavelengths longer than the X-ray band, where the effect of the density becomes more important.

As more data on short bursts gather, the information on the fraction of bursts as a function of the galaxy type will become an important element of study in order to establish whether there is a dominant channel of binaries (i.e. short-lived versus long-lived) that give rise to short bursts. However, if one wants to extract from these types of statistical studies meaningful physical information, one needs to keep in mind that there is a huge potential for selection biases. In fact, since bursts are localized through afterglow observations, and bursts occurring in elliptical galaxies are expected to be generally dimmer, there is going to be a selection effect toward the observation of a relatively larger fraction of the bursts occurring in starburst and spiral galaxies, i.e. the short-lived component of the binary population. Furthermore, since the mean redshift of merger events for the short-lived population is higher than that for the long-lived population, the selection effect described above will also result in a bias toward higher redshifts.

\subsection{The final moments: from a merging binary to a hyperaccreting disk around a black hole}

Although there is no "direct" evidence for an accretion disk in GRBs, the GRB phenomenology provides strong hints in that direction. Firstly, accretion disks are a powerful way to tap gravitational energy and channel it into other sources. Second, the overall (short) burst durations, as discussed above, are naturally accounted by the viscous timescale of the disk, while the millisecond timescale variability (observed both in long and short bursts) is on the same order of the dynamical timescale of a compact disk accreting around a stellar-mass black hole. From an observational point of view, on the other hand, it is well known that other systems in nature believed to be associated with black holes accreting from a disk (i.e. active galactic nuclei, micro-quasars) are able to power relativistic jets, for which we have strong evidence also in GRBs.

In the last several years, a number of groups have devoted a substantial effort into simulations of NS-NS and NS-BH mergers, and the resulting structure of the hyperaccreting accretion disk. The early simulations [?] were Newtonian, used a polytropic equation of state, and did not include the effects of neutrinos. These, on the other hand, were shown to represent a substantial source of cooling in a number of semianalytical, 1D calculations of hyperaccreting disks around black holes [59, 20] . Neutrino effects, together with more realistic equations of state, were taken into account into later simulations $[73,74,75,76]$. These simulations, independently of the nu- 
merical method used, found results in general agreement. Although details differ depending on whether the initial progenitor is a NS-NS or a NS-BH, some common features can be identified. As the binary members spiral in, within a few orbital periods the outcome is the formation of a dense and thick, hot torus, of mass on the order of $\sim 0.01-0.3 M_{\odot}$ that accretes onto a stellar mass black hole. In the case where the initial binary members are both neutron stars, the black hole will be formed as a result of the accretion of mass onto one of the NSs. Depending on the total initial mass of the binary system, the collapse of the hypermassive NS into a BH can occur promptly, or it can be delayed for an initial time during which the star is supported by differential rotation. The accreting material, on the other hand, is provided by the tidally disrupted debris of the NS.

The duration of the prompt GRB phase phase is set by the time during which efficient accretion occurs. Given the observed $\gamma$-ray luminosities $(\sim$ $10^{49}-10^{50} \mathrm{erg} / \mathrm{s}$ assuming a beaming correction of a factor $\sim 0.1$ ), and taking an efficiency of conversion of accretion energy into $\gamma$-rays of a fraction of percent to a percent, the accretion rates of the disk must then be in the range $\sim 0.01-10 M_{\odot}$. The resulting hyperaccreting disk is very dense and hot, optically thick to photons, and cools mainly by neutrinos. In the upper end of the range of accretion rates, the density of the disk can however be so high that the innermost regions would become optically thick even to the neutrinos themselves.

An important component of the studies of GRB accretion disks deals with the processes by which the disk black-hole system is able to collimate and launch the relativistic jets known to power the GRBs. Two mechanisms have been suggested as being involved: neutrino-anti neutrino annihilation, and magnetic fields. In the former process, suggested by a number of authors $[33,21,60,53,55]$, the source of energy is provided by the neutrinos and anti-neutrinos emitted in the cooling disk which annihilate in a funnel above a disk (which has a lower density). Calculations [?] have estimated that the maximum efficiency by which the rest mass energy of the accreting material is converted into neutrino luminosity does not exceed a value of $\sim 10^{-4}$. For a disk mass of $\sim 0.1 M_{\odot}$, this would yield an energy output of about $10^{49}$ erg, therefore making this jet-production mechanisms viable only if there is a substantial degree of collimation in short bursts.

The second method of jet collimation and launch relies on the help of magnetic fields. A number of authors [?, 84, 50] have suggesting this possibility by noticing how, even if the magnetic field is initially low, it is likely to be amplified by the magneto-rotational instability within the disk [2]. Numerical simulations [51] find that magnetically driven jets, whose energy output increases with the spin of the $\mathrm{BH}$, are generally more efficient than neutrinopowered jets. Magnetic fields are therefore considered to play an important role in collimating and driving the jets from the accretion disk.

Whereas a substantial progress has been made in this area of research of GRB hyperaccreting accretion disks, recent, new observations with Swift 
have shown that the current picture is far from being complete. In particular, the detection of energetic X-ray flares superimposed on the smooth afterglow decay, with arrival times and durations from tens to tens of thousands of seconds, requires the presence of an engine with duration much longer than the fraction of a second that is sufficient to power the prompt phase of a short burst [49]. These observations have prompted a number of suggestions on how to make the lifetime of the accretion disk much longer than its viscous timescale. Perna, Armitage \& Zhang ([67]; see also Piro \& Phfal [68]) suggested that fragmentation of the outer parts of the accretion disk (which would then accrete at later times) could be responsible for creating a longlived engine. Proga \& Zhang [71], on the other hand, envisaged a scenario in which the accumulation of magnetic flux in the innermost parts of the accretion disk creates a barrier that can then produce intermittent accretion. Other suggestions, which do not involve accretion disks, include that of Dai et al. [18]. They showed that, if the NS-NS merger leads to a differentially rotating, millisecond pulsar, then the differential rotation can cause the interior magnetic field to wind up and break through the stellar surface, hence resulting in magnetic reconnection-driven explosive events. These events would be observed as X-ray flares.

\section{Gravitational waves from short GRBs}

Mergers of two compact objects have traditionally been of great interest as sources of gravitational waves. With the likely association of binary mergers with short GRBs, the interest of the gravitational wave community has been extended to that of short GRBs. The local rate of these sources, however, is not large enough to make a detection likely with a blind search with LIGOI. However, the detection probability can be increased if the observations are made shortly after the $\gamma$-ray detection from the burst is detected. Estimates [43] suggest that in this case a positive detection in coincidence with a short GRB could be already made with current gravitational wave detectors. Clearly, such a signal, besides giving information on the last moments of the binary merger, would also provide the still needed conclusive evidence of the association of short bursts with mergers of compact objects.

\section{References}

1. Amati L., Della Valle M., Frontera F., Malesani D., Guidorzi C., Montanari E., Pian E., 2007, A\&A, 463, 913

2. Balbus, S. A. \& Hawley, J. F. 1991, ApJ, 376, 214

3. Band D., et al., 1993, ApJ, 413, 281

4. Belczynski, C., Bulik, T, \& Kalogera, V. 2002, ApJ, 571, 147 
5. Belczynski, K., Perna, R., Bulik, T., Kalogera, V., Ivanova, N. \& Lamb, D. Q. 2006, ApJ, 648, 1116

6. Berger E., et al., 2005, Nature, 438, 988

7. Berger E., Shin M. S., Mulchaey J. S., Jeltema T. E., 2006, ApJ submitted (astro$\mathrm{ph} / 0608498)$

8. Bloom, S. J., Sigurdsson, S. \& Pols, O. 1999, MNRAS, 305, 763

9. Bloom J. S., et al., 2006, ApJ, 638, 354

10. Brown, G. E. 1995, ApJ, 440, 270

11. Burgay, M. et al. 2003, Nature, 426, 531

12. Cameron P. B., et al., 2005, Nature, 434, 1112

13. Campana S., et al., 2006, A\&A, 454, 113

14. Castro-Tirado A. J., et al., 2005, A\&A, 439, L15

15. Christensen L., Hjorth J., Gorosabel J., 2004, A\&A, 425, 913

16. Costa E., et al., 1997, Nature, 387, 783

17. Covino S., et al., 2006, A\&A, 447, L5

18. Dai, Z. G., Wang, Z. Y., Wu, X. F., Zhang, B. 2006, Science, 311, 1127

19. Della Valle M., et al., 2006, Nature, 444, 1050

20. Di Matteo, T., Perna, R., \& Narayan, R. 2002, ApJ, 579, 706

21. Eichler, D., Livio, M., Piran, T., Schramm, D. N. 1989, Nature, 340, 126

22. Fruchter A. S., et al., 2006, Nature, 441, 463

23. Fox D. B., et al., 2005, Nature, 437, 845

24. Fryer, C., Burrows, A. \& Benz, W. 1998, ApJ, 496, 333

25. Fryer, C., Woosley, S. E.\& Hartmann, D. H. 1999, ApJ, 526, 152

26. Fynbo J. P. U., et al., 2006, Nature, 444, 1047

27. Gal-Yam A., et al., 2006, Nature, 444, 1053

28. Gehrels N., et al., 2005, Nature, 437, 851

29. Ghirlanda G., Ghisellini G., Celotti A., 2004, A\&A, 422, L55

30. Ghirlanda G., Ghisellini G., Lazzati D., 2004, ApJ, 616, 331

31. Ghirlanda G., Magliocchetti M., Ghisellini G., Guzzo L., 2006, MNRAS, 368, L20

32. Goodman, J. 1986, ApJ, 308, L47

33. Goodman, J., Dar, A. \& Nussmov, S. 1987, ApJ, 314, L7

34. Gorosabel J., et al., 2006, A\&A, 450, 87

35. Grupe, D., et al., 2007, ApJ in press (astro-ph/0603773)

36. Haislip J. B., et al., 2006, Nature, 440, 181

37. Hjorth J., et al., 2003, Nature, 423, 847

38. Hjorth J., et al., 2005, Nature, 437, 859

39. Hjorth J., et al., 2005, ApJ, 630, L117

40. Hobbs, G., Lorimer, D. R., Lyne, A. G., Kramer, M. 2005, MNRAS, 360, 974

41. Kalogera, V. et al. 1994, ApJ, 601, L179

42. Kawai N., et al., 2006, Nature, 440, 184

43. Kochanek, C. S. \& Piran, T. 1993, ApJ, 417, L17

44. Kouveliotou C., Meegan C. A., Fishman G. J., Bhat N. P., Briggs M. S., Koshut T. M., Paciesas W. S., Pendleton G. N., 1993, ApJ, 413, L101

45. Kulkarni S. R., et al., 1999, Nature, 398, 389

46. Lazzati D., Ramirez-Ruiz E., Ghisellini G., 2001, A\&A, 379, L39

47. Lazzati D., Begelman M. C., 2005, ApJ, 629, 903

48. Lazzati D., Ghirlanda G., Ghisellini G., 2005, MNRAS, 362, L8

49. Lazzati, D. \& Perna, R. 2007, MNRAS in press, astro-ph/0610730

50. Lyutikov, M. 2006, in AIP Conf. Proc. 838: Gamma-Ray Bursts in the Swift Era, ed. S. S. Holt, N. Gehrels and J. A. Nousek, 483

51. McKinney, J. C. \& Gammie, C. F. 2004, ApJ, 611, 977

52. Mereghetti S., Götz D., von Kienlin A., Rau A., Lichti G., Weidenspointner G., Jean P., 2005, ApJ, 624, L105

53. Meszaros, P. \& Rees, M.J. 1992, ApJ, 397, 570 
54. Metzger M. R., Djorgovski S. G., Kulkarni S. R., Steidel C. C., Adelberger K. L., Frail D. A., Costa E., Frontera F., 1997, Nature, 387, 878

55. Mochkovitch, R., Hernanz, M., Isern, J., Loisean, S. 1995, A\&A, 293, 803

56. Nakar E., Piran T., 2002, MNRAS, 330, 920

57. Nakar E., Gal-Yam A., Piran T., Fox D. B., 2006, ApJ, 640, 849

58. Nakar E., 2007, Physics Reports (astro-ph/0701748)

59. Narayan, R., Kumar, P. \& Piran, T. 2001, ApJ, 557, 949

60. Narayan, R. Paczynski, B. \& Piran, T. 1992, ApJ, 395, L83

61. Norris J. P., Marani G. F., Bonnell J. T., 2000, ApJ, 534, 248

62. Ofek, E., O., 2006, ApJ in press (astro-ph/0611860)

63. Paczynski, B. 1986, ApJ, 308, L43

64. Palmer D. M., et al., 2005, Nature, 434, 1107

65. Pedersen K., et al., 2005, ApJ, 634, L17

66. Perna, R. \& Belczynski, C. 2002, ApJ, 570, 252

67. Perna, R., Armitage, P. J. \& Zhang, B. 2006, ApJL, 636L, 29

68. Piro, A. L. \& Pfhal, E. 2006, ApJL in press, astro-ph/0610696

69. Popov S. B., Stern B. E., 2006, MNRAS, 365, 885

70. Portegies Zwart, S., F. \& Yungelson, L. R. 1998, A\&A, 332, 173

71. Proga, D. \& Zhang, B. 2006, MNRAS, 370, L61

72. Rhoads J. E., 1999, ApJ, 525, 737

73. Rosswog, S. et al. 1999, A\&A, 341, 499

74. Rosswog, S. \& Davies, M. B. 2002, MNRAS, 334, 481

75. Ruffert, M. \& Janka, H.-T. 1998, A\&A, 338, 535

76. Ruffert, M. \& Janka, H.-T. 1999, A\&A, 380, 544

77. Sari R., Piran T., Halpern J. P., 1999, ApJ, 519, L17

78. Soderberg A. M., et al., 2006, ApJ, 650, 261

79. Stanek K. Z., et al., 2003, ApJ, 591, L17

80. Tagliaferri G., et al., 2005, A\&A, 443, L1

81. Tanvir N. R., Chapman R., Levan A. J., Priddey R. S., 2005, Nature, 438, 991

82. Taylor G. B., Frail D. A., Beasley A. J., Kulkarni S. R., 1997, Nature, 389, 263

83. Terasawa T., et al., 2005, Nature, 434, 1110

84. Thompson, C. 1994, MNRAS, 270, 480

85. Tominaga N., Maeda K., Umeda H., Nomoto K., Tanaka M., Iwamoto N., Suzuki T., Mazzali P. A., 2007, ApJ, 657, L77

86. Tutukov, A. V. \& Yungelson, L. R. 1994, MNRAS, 268, 871

87. van Paradijs J., et al., 1997, Nature, 386, 686

88. Villasenor J. S., et al., 2005, Nature, 437, 855

89. Zhang B., Zhang B.-B., Liang E.-W., Gehrels N., Burrows D. N., Mészáros P., 2007, ApJ, 655, L25 